\title{
Use of a pancreatic stent as a guide for endoscopic ultrasound-guided fine-needle aspiration of a solid pancreatic mass lesion in a patient with underlying chronic pancreatitis
}

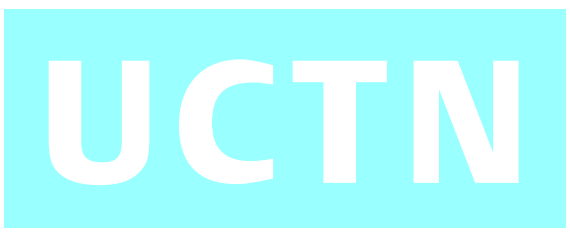

Endoscopic ultrasound-guided fine-needle aspiration (EUS-FNA) has a lower sensitivity for pancreatic mass lesions in the setting of chronic pancreatitis [1,2]. The EUS appearances associated with chronic pancreatitis limit imaging and therefore the interpretation of the changes seen with pancreatic masses: echo-poor foci in the pancreas make it difficult to decide on which area to puncture, and calcifications lead to acoustic shadowing that can conceal a mass. In addition, cytological interpretation can be challenging because the inflammatory infiltrate can obscure or simulate a pancreatic malignancy. We describe here a patient with underlying chronic pancreatitis in whom pancreatic stenting facilitated the diagnosis of pancreatic cancer at EUS-FNA.

A 68-year-old patient with history of chronic pancreatitis presented with abdominal pain and a 40-lb weight loss. Abdominal computed tomography and magnetic resonance cholangiopancreatography revealed chronic pancreatitis, without a focal mass. At EUS the pancreatic parenchyma was found to be echo-poor with lobulations, strading, and foci, and the main pancreatic duct was dilated and irregular, with side branches, findings consistent with chronic pancreatitis. These changes were distributed diffusely throughout the gland, with slightly more pronounced dilatation of the main pancreatic duct in the pancreatic body. FNA of this site revealed reactive ductal cells on a background of active inflammation (Figure 1). An endoscopic retrograde cholangiopancreatogram (ERCP) was then performed and this examination revealed two strictures: one in the neck region that was known previously to be benign, and another new stricture at the body-tail junction (Figure 2). A 5-Fr, 10-cm pancreatic stent was placed so that the proximal portion of the stent was located at the stricture in the body-tail junction. On repeat EUS, a discrete, echo-poor area was visualized at the body-tail junction where the stent was positioned. Two pas-
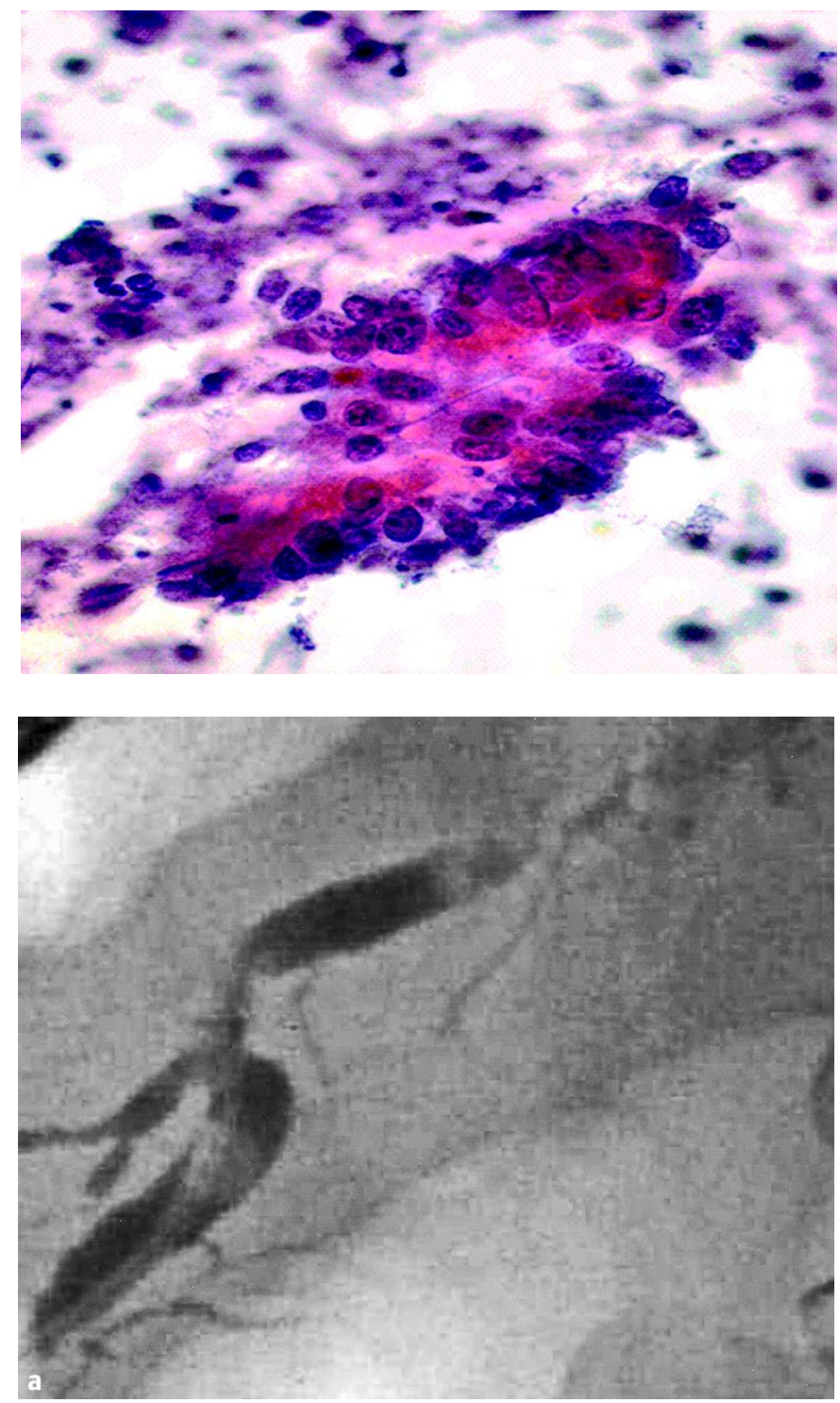

Figure 2 Pancreatography revealed two strictures, one at the neck that was known previously to be benign and another at the junction of the body and the tail of the pancreas (a). A stent was placed so that the proximal portion of the stent was located at the site of the stricture in the body-tail junction (b)

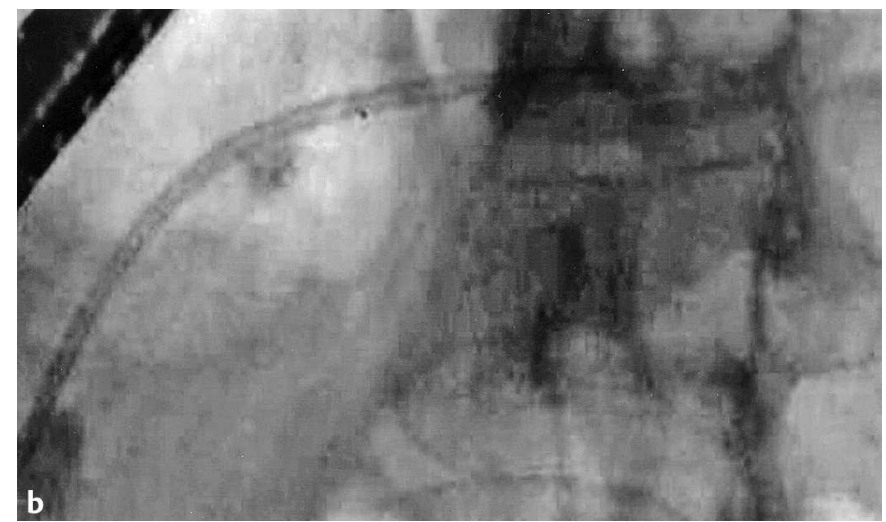




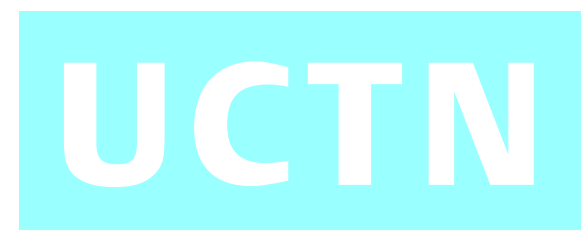

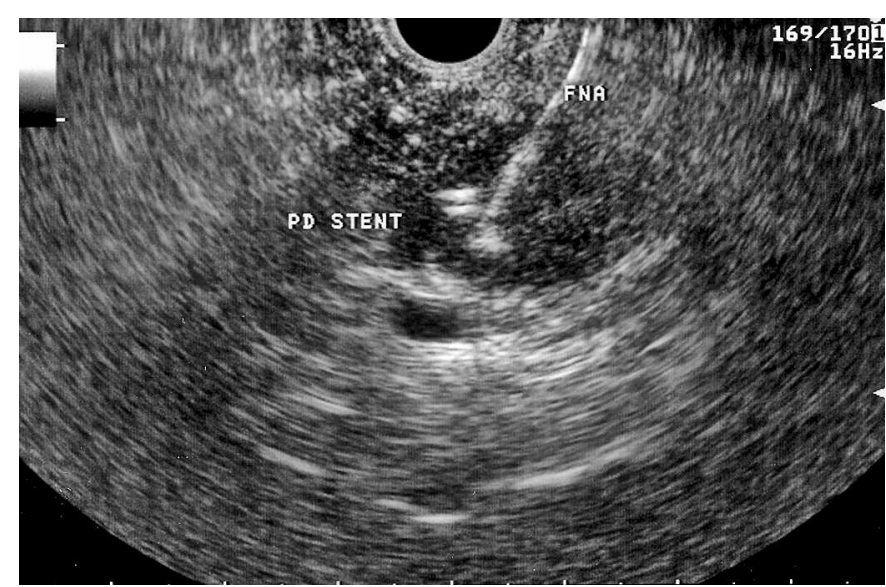

Figure 3 Repeat

EUS showed an echo-poor area that was now better visualized, adjacent to the stent in the body-tail junction of the pancreas, and this is where FNA was performed.

Figure 4 Histological view of material obtained at repeat EUS-FNA, showing findings consistent with a diagosis of pancreatic adenocarcinoma.

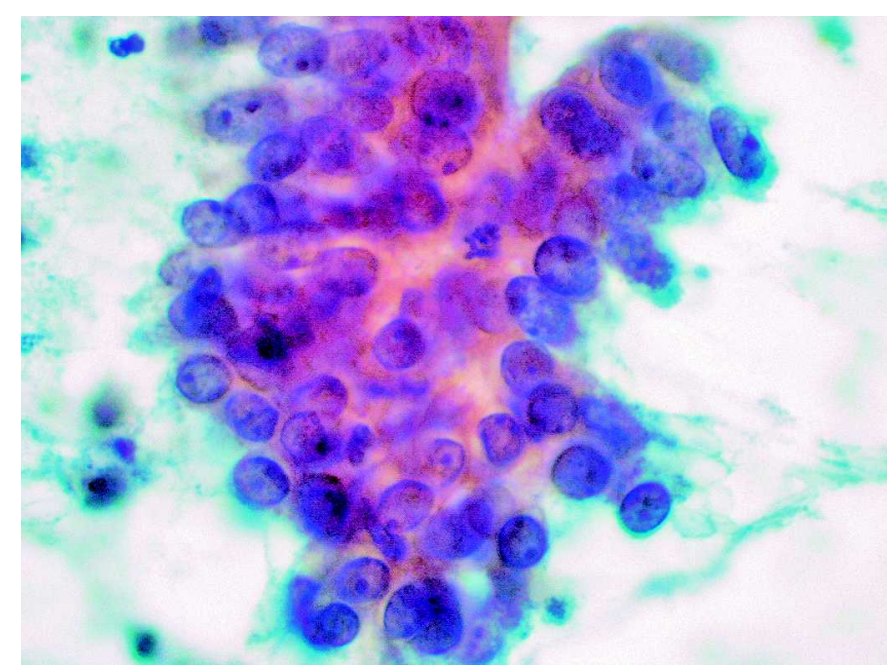

References

${ }^{1}$ Varadarajulu S, Tamhane A, Eloubeidi MA. Yield of EUS-guided FNA of pancreatic masses in the presence or the absence of chronic pancreatitis. Gastrointest Endosc 2005; 62: $728-736$

${ }^{2}$ Fritscher-Ravens A, Brand L, Knofel T et al. Comparison of endoscopic ultrasound-guided fine needle aspiration of focal pancreatic lesions in patients with normal parenchyma and chronic pancreatitis. Am J Gastroenterol 2002; 97: 2768-2775

${ }^{3}$ Erickson RA, Sayage-Rabie L, Beissner S. Factors predicting the number of EUS-guided fine needle passes for diagnosis of pancreatic malignancies. Gastrointest Endsoc 2000; 51: $184-190$

\section{Corresponding author}

\section{S. Varadarajulu, M.D.}

Division of Gastroenterology-Hepatology University of Alabama at Birmingham

Medical Center

410 LHRB, 1530 3rd Ave. S.

Birmingham

Alabama 35294

USA

Fax: $\quad+1-205-975-6381$

E-mail: svaradarajulu@yahoo.com ses were made at this site (Figure $\mathbf{3}$ ) and on-site cytopathological examination confirmed this to be a pancreatic adenocarcinoma (Figure 4). This diagnosis was subsequently confirmed at surgery.

Several investigators have reported falsenegative EUS-FNA investigations in the evaluation of solid pancreatic mass lesions in patients with underlying chronic pancreatitis because the needle can miss the lesion [3]. In this report we have shown that placement of a stent at the stricture enables better visualization of the mass and thereby improves the targeting of the FNA. This technique should be adopted only in the most challenging of circumstances, however, when other means of establishing the diagnosis have failed, because pancreatic stenting can induce post-ERCP pancreatitis.

Endoscopy_UCTN_Code_TTT_1AS_2AD

Endoscopy_UCTN_Code_TTT_1AS_2AF

\section{S. Varadarajulu1, N. C. Jhala ${ }^{2}$}

${ }^{1}$ Division of Gastroenterology-Hepatology, University of Alabama at Birmingham School of Medicine, Birmingham, Alabama, USA

2 Department of Pathology, University of Alabama at Birmingham School of Medicine, Birmingham, Alabama, USA. 